\title{
Influence of the Pulse Width for Visible Pulsed Laser Doping for Crystalline Solar Cells Using Phosphosilicate Glass
}

\author{
Malte SCHULZ-RUHTENBERG ${ }^{* 1}$, Anja HAEBERLE ${ }^{* 1}$, Richard RUSSELL ${ }^{* 2}$, José Luis HERNÁNDEZ ${ }^{* 3}$ and Stephan \\ KRANTZ $^{* 4}$ \\ ${ }^{* 1}$ Fraunhofer Institute for Laser Technology, Micro Technology, 52074 Aachen, Germany \\ E-mail: Malte.schulz-ruhtenberg@ilt.fraunhofer.de \\ ${ }^{* 2}$ BP Solar, 28108 Alcobendas (Madrid), Spain \\ ${ }^{* 3}$ IMEC, Process Technology Division, Leuven, 3001, Belgium \\ ${ }^{*}$ Solland Solar Cells BV, Heerlen, 6422, Netherlands
}

\begin{abstract}
To increase the conversion efficiency of silicon solar cells the creation of selective emitters is seen as an important step for improving existing production line cell efficiencies. By adding only one or a small number of additional processing steps into existing production lines, laser doping offers an attractive way of improving the cell efficiency significantly by $0.3-1 \%$ absolute. The selection of the proper laser parameters, such as wavelength, pulse length and laser power, allows control of the depth of the doping profile and the amount of laser-induced damage. This work deals with the possible processing parameters when using pulsed laser irradiation at $532 \mathrm{~nm}$ wavelength. The pulse width is varied in a range between 10 and $400 \mathrm{~ns}$. The analysis shows no direct influence of the pulse width on emitter depth of sheet resistance; these properties are governed by the laser power. However, the data indicates a larger process window for long pulses.
\end{abstract}

DOI:10.2961/jlmn.2011.01.0014

Keywords: crystalline solar cells, efficiency improvement, laser doping, pulse duration

\section{Introduction}

Photovoltaic (PV), the direct production of electrical current from sunlight, is one of the major hopes for a sustainable industrial society. This can be seen in the increase of cumulated installed PV power: from 2000 to 2008 it has increased tenfold from around 1,500 MW to nearly 15,000 MW [1]. The largest part, over $80 \%$, is made up by silicon wafer-based, so called "generation 1", solar cells.

To make PV competitive compared to conventional energy generation technologies it is imperative to reduce the main cost drivers, which are material, cell production and module production, each by about one third [2]. A good benchmark of the cost of a solar module is given by the electrical power generated by a standardized amount of sunlight divided by the module price (commonly expressed in Watt peak per dollar $\mathrm{Wp} / \$$ ). There are several possibilities to optimize this value, e.g. by increasing the productivity of the production chain, by reducing the amount of material used or by increasing the power generated by each cell. The latter is summarized by the cell's conversion efficiency. For commercial mono- and polycrystalline solar modules efficiencies lie between 12 and $17 \%$.

In the rapidly growing field of Photovoltaics, laser processing has become more and more important in recent years, because the promise of a contact-less, precise, high throughput laser tool that enhances solar cell production is an essential building block for a competitive sustainable energy supply. Laser selective emitter doping is one of five central laser processes developed within the EU funded project SOLASYS ("Next Generation Solar Cell and Module Laser Processing Systems").

Currently, the selective emitter concept is treated as one of the key processes for the improvement of existing solar cell manufacturing lines in the race to grid parity. Instead of using an intermediate doping concentration to meet both the demand for high carrier lifetimes and low contact resistance the doping concentration is increased locally at the positions of the front contacts. This can be achieved by laser doping the contact region before metal deposition.

\section{Motivation and Approach}

In the manufacturing process of wafer-based silicon solar cells the raw wafer is doped with phosphor to create the emitter layer and thus the essential pn-junction, which is responsible for the electrical function of the cell. The emitter is only 200 to 800 nanometers thick to allow sunlight to reach the junction. In today's production a trade-off between conductivity of the silicon and the life-time of the minority carriers is necessary, the former requiring a high, the latter a low doping concentration. Using selectively controllable doping profiles for the emitter the cell efficiency can be improved. Starting with a weakly doped emitter the doping concentration is increased locally at the positions of the metal contacts. Several methods to achieve selective doping exist, some of which include several chemical steps $[3,4]$ or a combination of laser and chemical processing[5]. Here a one-step method using direct laser beam treatment is presented. 


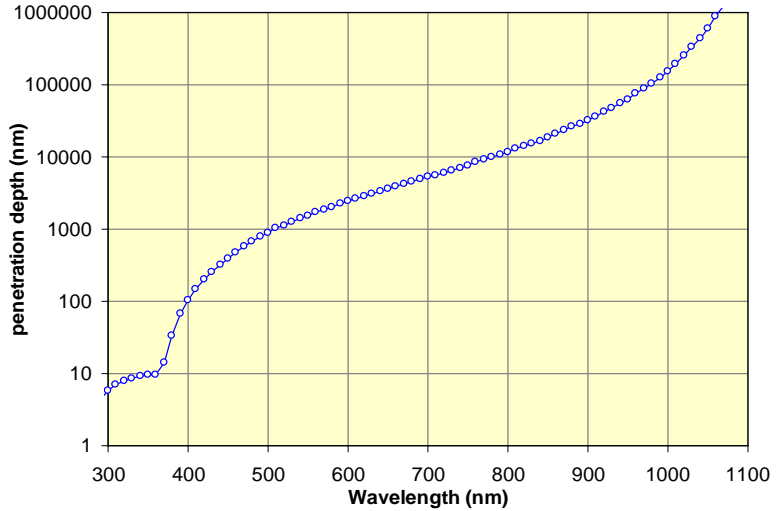

Figure 1: Dependence of the penetration depth in silicon on the irradiation wavelength.

For this one step doping process the phosphosilicate glass layer (referred to as PSG), which remains on the wafer surface after emitter formation and which is normally etched away, is used as dopant source. The silicon is locally heated respectively molten using laser irradiation, allowing the phosphor atoms to diffuse into the material. After irradiation the silicon cools down and re-crystallizes, enabling the phosphor atoms to enter the silicon lattice as donors. Depending on the required doping profiles, different laser wavelengths with suitable penetration depths and process adapted temporal irradiation profiles can be used. The great advantage of this efficiency increasing concept is the possibility to include the laser doping step into existing PV manufacturing lines, without increasing the complexity of the fabrication significantly.

In recent studies often a wavelength of $532 \mathrm{~nm}$ is used for laser doping, e.g. in $[6,7,8]$. IR and UV lasers are used only rarely $[\mathbf{9}, \mathbf{1 0}]$. This can in part be explained by the magnitude of the penetration depth, which of the order of 1 micrometer for $532 \mathrm{~nm}$, i.e. is comparable to the emitter depths (see figure 1 [11]). Pulse duration is normally in the

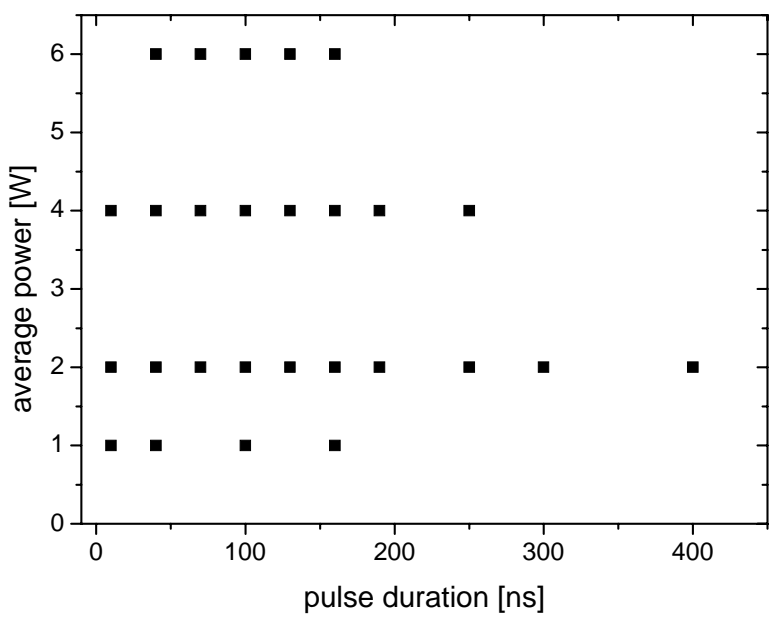

Figure 2: Accessible parameter range of the used laser. Each black dot corresponds to one parameter set used for the laser doping trials. The laser power is limited for shortest and longest pulse durations by the laser design.

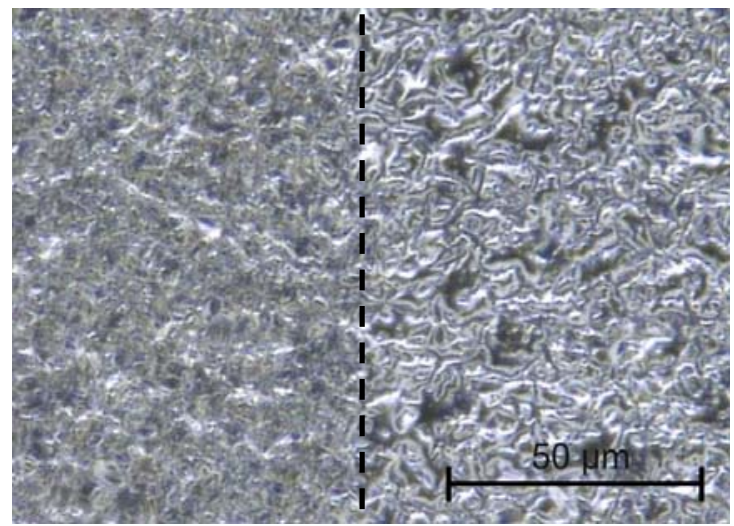

Figure 3: Micrograph of the border of a laser-treated region (marked by dashed line). Left side: untreated; right side: irradiated with 100 ns pulses, 6 Watt average power.

nanosecond range [12, 13], with some efforts using cw lasers [14]. However, there is no study available which illustrates the effect of the pulse duration on the doping profile in detail. This work tries to fill this gap in a first step for green laser radiation by varying the pulse duration from 10 to $400 \mathrm{~ns}$ and analyzing the resulting laser doped emitter by ECV (electrochemical capacitance-voltage) and sheet resistance measurements.

\section{Experiments}

The laser source used for the following experiments was provided by the Canadian company PyroPhotonics via the distributor Soliton. The PyroFlex 10-532 emits around $532 \mathrm{~nm}$ with a maximum power of 10 Watts and allows the selection of pulse duration, temporal pulse shape, repetition rate and pulse energy independently within a certain range. This promising new laser device was used to irradiate polycrystalline silicon wafers with a $60 \mathrm{Ohm} / \mathrm{sq}$ emitter and the residual PSG layer on top. The sample was positioned slightly out of focus so that an elliptical beam with Gaussian profile of about $50 \mu \mathrm{m}$ by $20 \mu \mathrm{m}$ was incident on the surface. Using a galvanometer scanner to deflect the beam (along the short axis of the ellipse) squares of 20 by 20 $\mathrm{mm}^{2}$ where created with a pulse overlap of around $10 \%$ in both directions. This ensures that only a fraction of the area is irradiated multiple times. However, parts of the irradiated areas are not covered by the laser pulses due to the elliptical shape. The effects of both the Gaussian beam profile and the elliptical beam shape are not clarified up to now. Pulse duration and average power could be varied in the range shown in figure 2, while the repetition rate was kept constant at $64 \mathrm{kHz}$ and the scanning speed at $3 \mathrm{~m} / \mathrm{s}$.

Figure 3 shows a microscope photograph of the border of a laser treated region. On the left the typical irregular surface of a polycrystalline wafer is shown. On the right the laser treatment can be seen in the form of features similar to beads forming on the surface due to melting of the silicon. The individual laser spot are not discernible, since the laser power was selected so that no ablation takes place.

The doped samples were characterized using an ECV measurement station to determine the doping profiles and several devices for the measurement of the sheet resistance: 


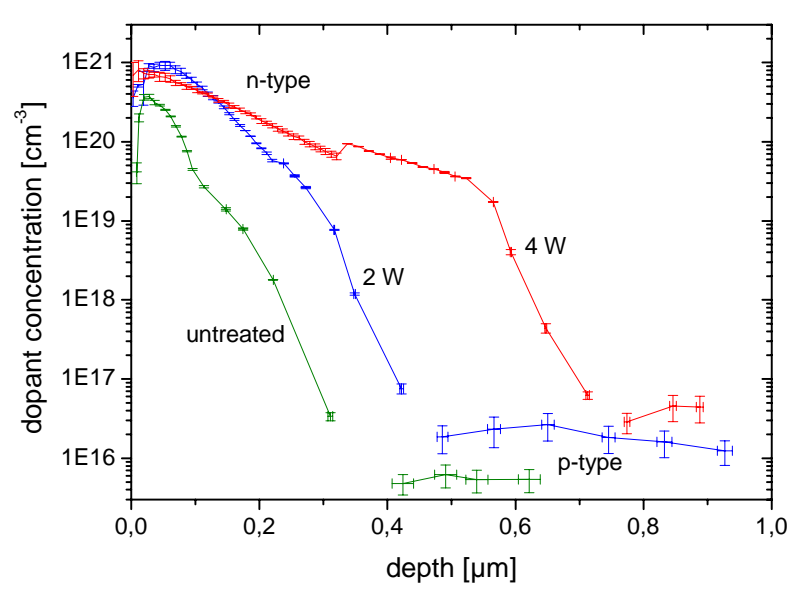

Figure 4: Emitter profiles measured with the ECV device. The green curves correspond to the original full-area emitter. Red and blue curves where doped with 40 ns pulses at two average powers.

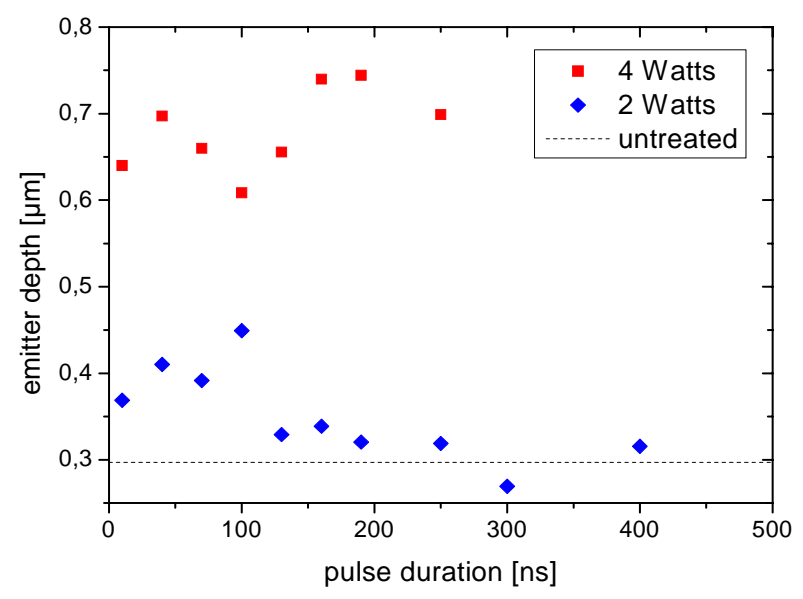

Figure 5: Emitter depth for various laser doped samples determined from ECV profiles. The dashed line corresponds to the original full-area emitter.

a handheld MR-1 resistance meter, a stationary Sherescan device and the ECV station. Since all sheet resistance measurements yield approximately the same values, the Sherescan values are presented in the graphs.

Figure 4 shows three selected ECV profiles, which show the concentration of n-type and p-type dopants plotted over the depth. The green curves correspond to the untreated sample areas and show the doping profile of the standard $60 \mathrm{Ohm} / \mathrm{sq}$ full area emitter applied to the wafers. Red and blue curves correspond to areas irradiated with 40 ns pulses and 2, respectively 4 Watts average power. Two important features are observed in the curves: the amount of active donors in the emitter is increased by the laser treatment from a maximum of $4 \cdot 10^{20} \mathrm{~cm}^{-3}$ to $1 \cdot 10^{21} \mathrm{~cm}^{-3}$ and the depths of the emitter increases strongly.

This latter effect is illustrated in more detail in figure 5, where the emitter depth is plotted over the pulse duration for 2 and 4 Watts average power, respectively. The values of the emitter depth were determined by fitting a line to the last three data points of the n-type region of the ECV pro-

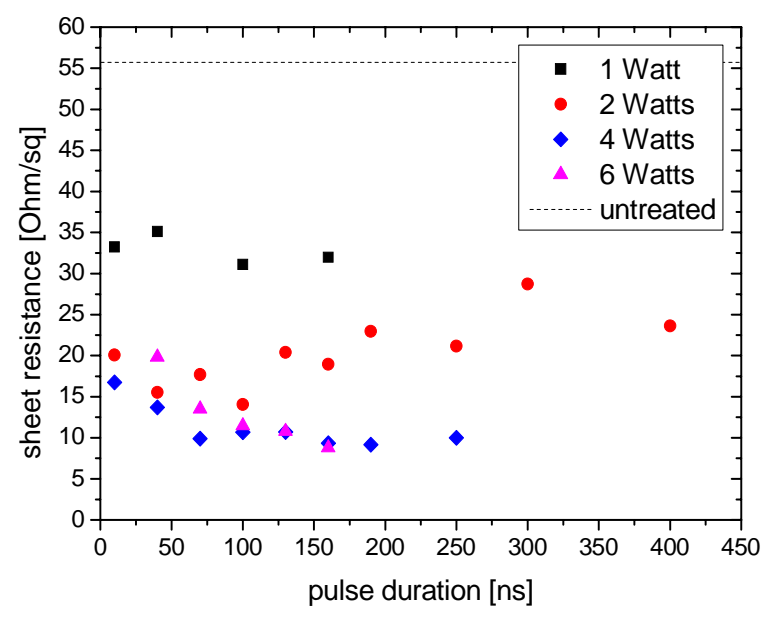

Figure 6: Dependence of sheet resistance on pulse duration for different average powers. The dashed line corresponds to the original full-area emitter.

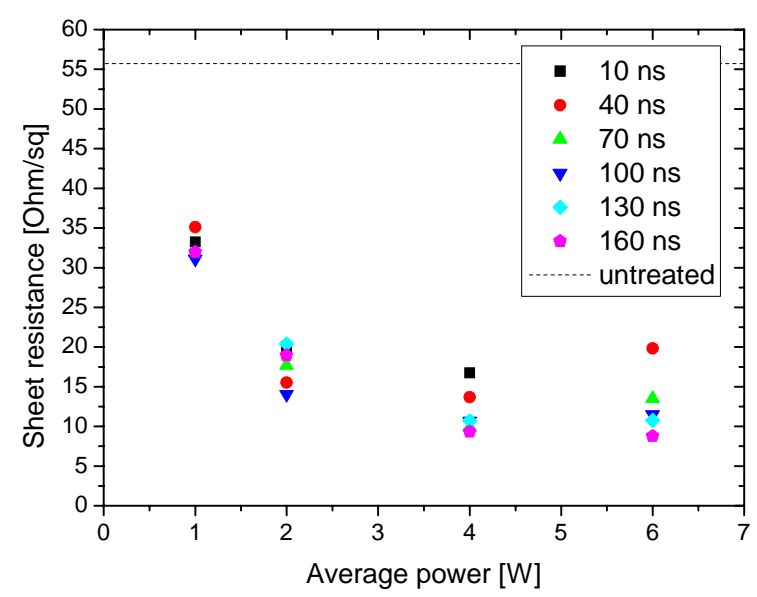

Figure 7: Dependence of sheet resistance on average power for different pulse durations. The dashed line corresponds to the original full-area emitter.

files and calculating the intersection with the p-type background doping of about $1 \cdot 10^{16} \mathrm{~cm}^{-3}$. The influence of the surface roughness of a few micron on the ECV measurements was not considered here, though it may be responsible for the large fluctuations in the data. The measurement of the reference full area emitter yielded the correct depth of $\sim 300 \mathrm{~nm}$. The dashed line corresponds to the depth of the untreated emitter $(300 \mathrm{~nm}$ ) and it is again observed that laser treatment increases the emitter depth. However, a strong dependence on the pulse duration is not evident. A strong influence of the average power is observed, but due to limited resources for ECV measurements here only two different average powers are plotted.

To further analyze the effect of the laser doping process the sheet resistance was measured, which is a measure for the doping concentration within the whole emitter layer. Figure 6 and 7 illustrate the change of the sheet resistance, and thus - in first approach - the change of the doping concentration with the two laser parameters pulse duration and average power. Again the dashed line corresponds to the 
untreated reference emitter. A decrease of the sheet resistance to values as low as $10 \mathrm{Ohm} / \mathrm{sq}$ is evident.

\section{Discussion}

The experimental results show that emitter depth increases and sheet resistance decreases with average power, which was expected since the total energy deposited in the sample and thus the temperature increases. Also, due to thermal diffusion, a larger volume of the material is heated. Laser doping occurs for all laser parameters in the range selected for the experiments. Within the average power range no ablation was observed in the microscopic images. Also a strong increase in sheet resistance, indicating the ablation of the emitter layer [9], was not observed.

However, a strong dependence on pulse duration is not evident in the range under study. Here, it was expected that a longer duration of irradiation results also in a longer diffusion time and ultimately in a deeper emitter, which was indicated in [15]. The experiments described here demonstrate that this is not the case. Only a small decrease of the sheet resistance with pulse duration is observed in figure 6 for high average powers.

The question, which type of laser source is most suitable for laser doping can be answered tentatively: in figure 7 a weak increase of the sheet resistance for high average powers is visible for a pulse duration of $40 \mathrm{~ns}$. This can probably be attributed to the onset of material ablation. Also in figure 6 the strongest dependence of the sheet resistance on pulse duration is observed for the maximum power of 6 Watts. Altogether, this means that the data indicates a larger processing window in terms of suitable laser powers for longer pulse durations. If the pulsed irradiation at $532 \mathrm{~nm}$ wavelength is the most suitable process for laser doping has to be shown by further investigations.

The fluctuations in the data are probably due to the fact that polycrystalline material was used for these experiments. This is in part compensated by the large range of pulse durations covered by the experiments. However, a much stronger evidence for the trends described here would probably be gained from the same experiments conducted on monocrystalline silicon samples.

\section{Industrial scale-up}

In addition to the physical characteristics of the selectively doped emitter it is important to keep the industrial feasibility in mind. Of course, emitter and metallization strongly interact, so it is important to distinguish two different cases: a) doping of the finger pattern only and b) doping of busbars and fingers. The former can be applied to screen-printed or lithographically printed contacts, the latter to self-aligning plated contacts. For both cases 156 $\mathrm{mm}$ wafers and 60 fingers are assumed.

For case a) the doped lines below the fingers have to be wide enough to securely place the full metal contacts onto the doped area. A finger width of $200 \mu \mathrm{m}$ is thus assumed. This means that with a $30 \mu \mathrm{m}$ laser spot size on the sample 9 passes are needed per finger, resulting in a total pass length of nearly 84 meters. With $64 \mathrm{kHz}$ and $10 \%$ spot overlap a scan speed of $1.7 \mathrm{~m} / \mathrm{s}$ is required. The total process time amounts to about 54 seconds per wafer, much too slow for industrial applications. With a repetition rate of $500 \mathrm{kHz}$ a scanning speed of demanding $13.5 \mathrm{~m} / \mathrm{s}$ is re- quired, resulting in a processing time of less than 7 seconds per wafer. If the spot size is increase to $80 \mu \mathrm{m}$ (which means that the pulse energy has to be increase accordingly to keep the fluence constant) the repetition rate has to be reduced to $250 \mathrm{kHz}$ to keep the scanning speed below 20 $\mathrm{m} / \mathrm{s}$. This results in a processing time of 1.7 seconds. However, for such a process the laser source would be required to deliver at least 110 Watts average power, which is beyond the capabilities of current solid state lasers. Advances in screen-printing which allow narrower fingers would make this process industrially feasible.

For case b) two busbars of $1 \mathrm{~mm}$ width are assumed, the fingers can be as narrow as $50 \mu \mathrm{m}$, supposed the plating process allows such narrow fingers. In this case only two passes per finger are required. The pass length would amount to 19 meters for the fingers and 13 meters for the busbars. With the laser used here the processing time would amount to about 20 seconds. Scaling up to $350 \mathrm{kHz}$ and a $50 \mu \mathrm{m}$ spot size the processing time would be reduced to only 1.2 seconds at a scanning speed of $16 \mathrm{~m} / \mathrm{s}$. Still, 60 Watts laser power would be required. If the selective emitter concept is combined with plated contacts, this could be a feasible process for industrial application, but it is quite obvious that with existing commercial laser sources more than one machine would be required to reach the processing times of 1 second per wafer.

\section{Conclusion and Outlook}

This study investigates the influence of two essential laser parameters, namely average power and pulse duration, on the emitter characteristics during laser doping. Using a very flexible laser source emitting at $532 \mathrm{~nm}$ the pulse duration was varied from 10 to 400 ns and the laser power from 1 to 6 Watts. The resulting laser doped samples were analyzed by ECV measurements to determine the emitter depth. A strong dependence of this depth on the average power was observed while the influence of the pulse duration was found to be insignificant. By analyzing sheet resistance measurements it was demonstrated that the pulse duration has a minor influence on the total amount of active dopants as well. A beginning increase of the sheet resistance for short pulses and high average power indicates that longer pulses allow a larger process window.

For an industrialization of the laser doping process an estimation of the processing time was calculated for different scenarios. Here, it can be concluded that with commercial lasers and state-of-the-art metallization processes a laser doping machine could be competitive in terms of throughput if at least two machines work in parallel.

For a comprehensive success the promising selective emitter concept requires more basic investigations from the point of view of the laser specialists. In addition to the study presented here, it is imperative to look at other wavelengths like near IR and UV in a similar approach. Our group is planning to use monocrystalline material for further studies to eliminate any uncertainties in the data arising from the sample itself. Also, for a study more closely related to the selective emitter application samples should be prepared with a weakly doped emitter of around 100 $\mathrm{Ohm} / \mathrm{sq}$. The effects of a Gaussian beam profile and a nonuniform irradiation due to a circular or elliptical beam shape should be investigated in future studies. 


\section{Acknowledgments}

The work presented has been partially funded by the European Commission within the project "SOLASYS" in the 7th Framework programme. The authors would like to thank the European Commission and the project partners for their contribution.

Special thanks go to PyroPhotonics (Québec, Canada) for lending the laser source, to the team of Rubin Sidhu at BP Solar Frederick (Maryland, USA) for the ECV measurements and to Robert Bleidiessel at Solland Solar Cells (Netherlands) for the Sherescan measurements.

\section{References}

[1] European Photovoltaic Industry Association EPIA. Global market outlook until 2013. www.epia.org, April 2009.

[2] C. del Canizo, G. del Coso, and W.C. Sinke. Crystalline silicon solar module technology: Towards the 1 euro per watt-peak goal. Prog. Photovolt: Res. Appl., 17(3):199-209, 2009.

[3] M. Lozach, R. Monna, Y. Veschetti, N. Enjalbert, M. Pirot, and A. Bettinelli. Selective emitter formation by pocl3 or 'spin on' source diffusion for industrial large scale mc-si solar cells. In Proceedings of 22nd European Photovoltaic Solar Energy Conference, 3-7 September 2007, Milan, Italy, 2007.

[4] R. Monna, N. Enjalbert, Y. Veschetti, M. Lozach, and M. Pirot. $17.8 \%$ efficiency obtained on c-si solar cells using a selective emitter industrial type process. In Proceedings of 23rd European Photovoltaic Solar Energy Conference, Valencia, Spain, 2008.

[5] Sybille Hopman, Andreas Fell, Kuno Mayer, Monica Aleman, Matthias Mesec, Ralf Mueller, Daniel Kray, and Gerhard P. Willeke. Characterization of laser doped silicon wafers with laser chemical processing. In 22nd European Photovoltaic Solar Energy Conference, 2007.

[6] C. Carlsson, A. Esturo-Breton, M. Ametowobla, J.R. Koehler, and J.H. Werner. Laser doping of textured monocrystalline silicon wafers. In 22nd European Photovoltaic Solar Energy Conference, 2007.
[7] B. S. Tjahjono, J. H. Guo, Z. Hameiri, L. Mai, A. Sugianto, S. Wang, and S. R. Wenham. High efficiency solar cell structures through the use of laser doping. In 22nd European Photovoltaic Solar Energy Conference, 2007.

[8] M. Ametowobla, J.R. Koehler, A. Esturo-Breton, and J.H. Werner. Improved laser doping for silicon solar cells. In 22nd European Photovoltaic Solar Energy Conference, 2007.

[9] M.C. Morilla, R. Russell, and J.M. Fernandez. Laser induced ablation and doping processes on high efficiency silicon solar cells. In 23rd European Photovoltaic Solar Energy Conference, 2008.

[10]Akiyoshi Ogane, Kenji Hirata, Koyo Horiuchi, Athapol Kitiyanan, Yukiharu Uraoka, and Takashi Fuyuki. Application of laser doping technique to bulk and thin film multicrystalline silicon solar cells. In 23rd European Photovoltaic Solar Energy Conference, 2008.

[11]M.A. Green and M. Keevers. Optical properties of intrinsic silicon at $300 \mathrm{k}$. Progress in Photovoltaics: Research and Applications, 3:189-192, 1995.

[12]M. Ametowabla, A. Esturo-Breton, J.R. Kohler, and J.H. Werner. Laser processing of crystalline silicon solar cells. In Photovoltaic Specialists Conference, 2005. Conference Record of the Thirty-first IEEE, pages 1277-1280, 2005.

[13]Ulrich Jaeger, Mahir Okanovic, Matthias Hoertheis, Andreas Grohe, and Ralf Preu. Selective emitter by laser doping from phosphosilicate glass. In Proceedings of 24th European PVSEC, Hamburg, Germany, 2009.

[14]Kenji Hirata, Akiyoshi Ogane, Takashi Saitoh, Athapol Kitiyanan, Emi Sugimura, and Takashi Fuyuki. Optimization of cw laser doping in crystalline silicon solar cell fabrication process. In Proceedings of 24th European PVSEC, Hamburg, Germany, 2009.

[15]Mahir Okanovic, Ulrich Jaeger, Marc Ahrens, Uwe Stute, Andreas Grohe, and Ralf Preu. Influence of different laser parameters in laser doping from phosphosilicate glass. In Proceedings of 24th European PVSEC, Hamburg, Germany, 2009.

(Received: June 07, 2010, Accepted: February 14, 2011) 